\title{
Bilateral polymicrogyria
}

INSERM

\section{Source}

INSERM. (1999). Orphanet: an online rare disease and orphan drug data base. Bilateral polymicrogyria. ORPHA:268940

Bilateral polymicrogyria is a rare cerebral malformation due to abnormal neuronal migration defined as a cerebral cortex with many excessively small convolutions. It presents with developmental delay, intellectual disability, seizures and various neurological impairments and may be isolated or comprise a clinical feature of many genetic syndromes. It may also be associated with perinatal cytomegalovirus infection. 